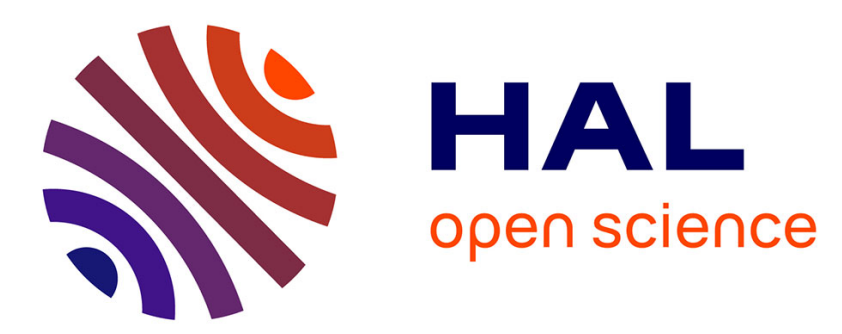

\title{
Strong coupling between pyramidal cell activity and network oscillations in the olfactory cortex.
}

\author{
P. Litaudon, S. Garcia, N. Buonviso
}

\section{To cite this version:}

P. Litaudon, S. Garcia, N. Buonviso. Strong coupling between pyramidal cell activity and network oscillations in the olfactory cortex.. Neuroscience, 2008, 156 (3), pp.781-7. 10.1016/j.neuroscience.2008.07.077 . hal-00350375

\section{HAL Id: hal-00350375 https://hal.science/hal-00350375}

Submitted on 6 Jan 2009

HAL is a multi-disciplinary open access archive for the deposit and dissemination of scientific research documents, whether they are published or not. The documents may come from teaching and research institutions in France or abroad, or from public or private research centers.
L'archive ouverte pluridisciplinaire HAL, est destinée au dépôt et à la diffusion de documents scientifiques de niveau recherche, publiés ou non, émanant des établissements d'enseignement et de recherche français ou étrangers, des laboratoires publics ou privés. 
Title: Strong coupling between pyramidal cell activity and network oscillations in the olfactory cortex

Litaudon, P., Garcia, S., Buonviso, N.

Neurosciences Sensorielles, Comportement, Cognition, CNRS UMR 5020 - Université

Lyon 1, Université de Lyon, Institut Fédératif des Neurosciences de Lyon, Lyon, France

Corresponding author : P. Litaudon, Neurosciences Sensorielles, Comportement, Cognition, Université Lyon I - CNRS, 50 avenue Tony Garnier, 69366 Lyon cedex 07, France Tel.: (33) 437287461

Fax: (33) 437287601

e-mail: litaudon@olfac.univ-lyon1.fr

Section Editor: Richard J. Weinberg 
Abbreviations

PC: piriform cortex

aPC (anterior piriform cortex)

OB: olfactory bulb

LFP: local field potential

I/E: inspiration/expiration 


\section{Abstract}

Oscillatory activity is a prominent characteristic of electrophysiological recordings in the olfactory system and has been proposed to play a key role in encoding olfactory representations. Studies in several systems have shown that some aspects of information coding involve characteristics that intertwine spikes and fast oscillations (in the beta and gamma range) of local field potentials (LFP). In the insect olfactory system, it has been proposed that oscillatory activity could provide a temporal link between cells. Following previous data, we have proposed that gamma band oscillations in mammals could subserve a gating function for the transfer of information between the olfactory bulb (OB) and the anterior piriform cortex (aPC), which are functionally coupled. In this study, we used an electrophysiological approach to investigate the temporal relationship between LFP gamma oscillations and single-unit activity by simultaneously recording LFP and single unit discharges in the aPC during odor evoked activity. Our data showed that mean spike discharges and gamma oscillatory bursts were synchronized with the same respiratory cycle epoch (around the inspiration/expiration transition). Temporal correlations between spikes and LFP revealed that cortical cell spikes were tightly phase-coupled with the peak of gamma oscillations and that this phase-coupling was not odor-dependent. Our results suggest that gamma oscillation may act as a temporal filter. Oscillatory phase-coupled spikes in the OB could act in increasing the probability of spike emission in the aPC cell during a narrow timewindow, explaining the tight phase-coupling observed in the aPC. The role of spike-LFP phase-coupling as a binding function between odor features is discussed.

\section{Key words:}

rat, piriform cortex, olfactory coding, local field potential, gamma oscillations 
Oscillatory activity is a prominent characteristic of electrophysiological activity in the olfactory system and has been well-described in the olfactory bulb (OB) and the insect antennal lobe. It has been proposed to play a key role in encoding olfactory representations (Freeman, 1975; Laurent and Davidovitz, 1994). This oscillatory activity consists of a slow modulation related to breathing and fast oscillations, in the beta range (15-35 Hz) and in the gamma range (40-80 Hz), related to network dynamic. In different sensory systems, it has been proposed that fast oscillatory activity could provide a temporal link between cells and could support cell synchronization (see Singer, 1999 for review). In the olfactory system, the temporal relationship between fast oscillations and neuronal spike discharges has been first studied in the insect antennal lobe (Laurent and Davidovitz, 1994; Laurent et al., 1996; PerezOrive et al., 2002). The authors proposed that phase-locking of single unit activity onto field potential oscillations could play a role in temporal linking of activities for odor-specific neuronal assemblies. Pharmacological desynchronization in honeybees impaired fine discrimination in a behavioral paradigm (Stopfer et al., 1997), demonstrating the functional relevance of oscillatory synchronization. In the fish $\mathrm{OB}$, it has been proposed that spikes phase-locked with the oscillations convey information about odor category, whereas non phase-locked spikes convey information about odor identity (Friedrich et al., 2004). In the mammalian $\mathrm{OB}$, the single report regarding the temporal relationship between fast oscillations and spikes (Kashiwadani et al., 1999) revealed that mitral/tufted cell spikes tended to phaselock with the oscillatory local field potential (LFP) and exhibited synchronized oscillatory spike discharges. The synchronization of spike discharges may increase the probability of driving the target neuron in the piriform cortex (PC), due to temporal summation of synaptic inputs onto pyramidal neurons. 
In the mammalian PC, fast oscillatory activity has also been described (Freeman, 1978; Bressler, 1987; Neville and Haberly, 2003). The only attempt to analyze correlations between unit firing and EEG activity has been performed during spontaneous activity without any odor stimulation (Eeckman and Freeman, 1990). The PC is a functionally heterogeneous structure (Litaudon and Cattarelli, 1994; Litaudon and Cattarelli, 1995; Litaudon et al., 1997; Neville and Haberly, 2003; Kadohisa and Wilson, 2006) characterized by a decrease in afferent activity along its antero-posterior axis (Haberly, 1973; Litaudon et al., 2003). Previous data suggested that pyramidal cells in the anterior PC (aPC) receive a large and synchronized excitation from the OB and that OB and aPC are functionally coupled (Chabaud et al., 1999; Litaudon et al., 2003). Regarding the simultaneous occurrence of gamma (40-80 Hz) rhythm (Neville and Haberly, 2003) in the OB and PC during odor sampling, we have proposed that gamma band oscillations could subserve a kind of gating function for bulbo-cortical transfer of information (Buonviso et al., 2003). If the OB and aPC are functionally coupled via gamma oscillations, bulbar output spikes should drive PC cells during a preferential phase of the gamma oscillation. As aPC neurons integrate input during narrow time windows (Franks and Isaacson, 2006), spikes evoked in cortical cells in response to odor should be phase-locked to the LFP gamma oscillation. A similar mechanism has been proposed in the insect mushroom body, which could be considered, to some extent, as the analog of the mammal olfactory cortex. It has been proposed that Kenyon cells act as coincidence detectors, the oscillatory dynamic acting as a selective filter to synthetize the output from the antennal lobe (PerezOrive et al., 2002).

To test this hypothesis, we investigated the temporal relationship between LFP gamma oscillation and unit activities in the aPC by simultaneously recording the LFP and single unit discharges. We observed strong phase-coupling in the anterior PC between pyramidal cell 
spikes and gamma oscillations. Such results suggest that gamma oscillations could provide an appropriate time-window for coincident spike detection in the aPC.

\section{Experimental procedures}

\section{Preparation and recording}

All procedures were conducted in strict accordance with the European Communities Council guidelines. Experiments were performed on 22 male Wistar rats (Charles River Labs, L’Arbresle, France), weighing 250-350 g. Preparation and recordings were carried out in naturally breathing, urethane $(1.5 \mathrm{~g} / \mathrm{kg})$ anesthetized rats. Anesthesia was maintained by supplemental doses when necessary. During the experiment, respiratory activity was monitored through a homemade airflow sensor placed near the entrance of the nostril (Roux et al., 2006).

Odors (isoamyl acetate (ISO), p-cymene (CYM), cineole (CIN), geraniol (GER), eugenol (EUG), anisole (ANI), d-limonene (LIM), and 2-heptanone (HEP)) were presented with a flow dilution olfactometer at a concentration of $\sim 2.10^{-1}$ of saturated vapor. Stimuli lasted $5 \mathrm{~s}$ and were separated from each other by at least $2 \mathrm{~min}$. Stimulus onset was triggered on the respiratory cycle (at the maximum of exhalation).

Extracellular single-unit activity and local field potentials were recorded from PC layer II/III neurons using glass micropipettes (7-12 M 2 ) filled with $0.5 \mathrm{M}$ sodium acetate saturated with pontamine sky blue. A bipolar concentric electrode was positioned in the OB mitral cell layer (MCL). Recording electrodes were lowered from the dorsal surface in the anterior PC $(1.5<\mathrm{AP}<2.5,3.5<\mathrm{ML}<4$ relative to Bregma). Placement in layer II/III was determined according to the shape of the field potential evoked by MCL electrical stimulation (Haberly, 1973; Litaudon et al., 2003). After the recording session, the location of the electrode tip was 
marked using dye spot iontophoretically deposited (2-5 $\mu \mathrm{A}$ for $10 \mathrm{~min}$ : 10s on, 10s off), and its placement in the PC was confirmed histologically.

Cortical activity was recorded as a broad-band signal $(0.1 \mathrm{~Hz}-5 \mathrm{kHz})$. Cortical and respiratory signals were sampled at $10 \mathrm{kHz}$ and acquired using the IOTech acquisition system (Wave Book).

\section{Data analysis}

All programs and routines were written and implemented in the Python programming language. Data obtained from respiratory signal, LFP and single unit activity analyses were stored in a MYSQL engine database.

Respiratory signal

In order to take into account the respiratory modulation of odor-evoked activity (Litaudon et al., 2003; Buonviso et al., 2006), data were represented as a function of respiratory phase. Respiratory signal processing presented here was a modified version of the analysis method described in a previous paper (Roux et al., 2006). The respiratory cycle was first divided into two periods: inspiration and expiration. Second, the mean duration of both periods was computed from all recordings. Third, inspiration and expiration periods of each recording were resized according to this mean duration, and the total length of the respiratory cycle was normalized to one (see figure 1B). Thus, this phase representation of the respiratory cycle was used as a normalized time basis (between 0 and 1) permitting us to collect and analyze results in a standardized data format across different recordings.

\section{Local Field Potentials}

\section{$\underline{\text { Wavelet transform }}$}

LFPs were obtained by band-passing the recorded signal at 5-200 Hz. Time and frequency information were obtained using a time-frequency representation (Fig. 1A2) based on continuous wavelet transform; the method has been described in detail by Roux et al. (2007). 
Briefly, the down-sampled LFP signal was convoluted with Morlet's complex wavelets (Kronland-Martinet et al., 1987). Morlet's wavelets stretch from 0 to $100 \mathrm{~Hz}$ with a $0.25 \mathrm{~Hz}$ resolution in the frequency domain and a $5 \mathrm{~ms}$ resolution in the temporal domain. To analyze respiratory modulation, time-frequency maps were first segmented into successive respiratory cycles with alternating inspiration and expiration windows. Each window was re-sampled according to the normalized respiratory cycle (see above). This phase-frequency representation was used to compute the mean representation of oscillatory activity (Fig. 1B) related to respiratory cycle (Roux et al., 2006).

\section{$\underline{\text { Wavelet ridge extraction }}$}

The main goal of this study was to analyze the correlations between spikes, fast transitory phenomena that occur at high frequency, and LFP oscillations, which are low frequency phenomena. Instantaneous phase and frequency of the oscillatory episodes were obtained using a new algorithmic procedure based on the wavelet ridges extraction method (see Roux et al., 2007 for details). Briefly, we computed the mean and standard deviation (STD) of the time-frequency representation and defined a threshold as the mean ( \pm 5 STD) of the timefrequency energy contained in the pre-stimulus period (between 0 and $10 \mathrm{sec}$ ). Then, the coordinates in time and frequency of all local maxima in the gamma band (40-80 Hz) above this threshold were extracted (Fig. 2A1). From each maximum, we computed a high timeresolution (0.1 ms) Morlet's complex wavelet transformation, both forwards and backwards, following the line of maximum energy. The computation stopped when the energy fell below the threshold. Therefore, for each maximum detected on the time frequency map, we obtained a high time-resolution wavelet ridge defined by its starting and ending times, its instantaneous frequency and instantaneous phase (Fig. 2A). 
Thus, each gamma episode was characterized by the coordinates of its maximum (amplitude, frequency, respiratory phase) and its wavelet ridge (duration, instantaneous phase and frequency).

\section{Single unit activity}

Single unit activity was extracted using a bandpass filter (300-3000 Hz). The single-unit nature of the recordings was confirmed with auto-correlograms showing at least a $3 \mathrm{~ms}$ refractory period. Each spike was time-stamped from the raw signal, and this time position was converted into a phase position relative to the respiratory cycle, which was used to compute a histogram of phase distribution along the respiratory cycle (Litaudon et al., 2003; Roux et al., 2006).

\section{Spike-LFP phase coupling.}

For each time-stamped spike that occurred during an oscillatory epoch, we marked the instantaneous phase of the LFP signal at that time stamp (Fig. 2A3) and then built its spike preferential instantaneous phase histogram (Fig. 2B) relative to gamma oscillatory activity.

\section{Statistical analysis}

The Rayleigh test for directionality was used in order to determine if spikes and gamma oscillations were uniformly distributed (unsynchronized) or non-uniformly distributed (synchronized) relative respiratory cycle (Litaudon et al., 2003). Comparisons of phase between odors were performed using a Watson $\mathrm{F}$ test (this statistical test is specific for circular data) (Mardia, 1972; Fisher, 1993). The same statistical tests were used to analyze phase relationships between spikes and oscillations of the LFP. 


\section{Results}

A total of 315 odor evoked responses obtained from 51 cells recorded in the aPC were analyzed in this study.

\section{Local Field Potentials}

We first characterized the frequency distribution of PC oscillatory activity using continuous wavelet transform. The result of the wavelet transform is illustrated as an energy scalogram, which represents the time-varying energy of the signal in each frequency band, leading to a time-frequency representation of the LFP signal. Spontaneous LFPs were generally fairly flat (Fig. 1A), and the following analysis will focus on the odor-evoked activity period. During odor-evoked activity, we observed a prominent gamma frequency oscillation $(40-80 \mathrm{~Hz})$ in the aPC and more irregular activity in the beta band (12-30 Hz). Gamma oscillations occurred in regular bursts synchronized with respiration. In order to visualize this respiratory modulation, phase-frequency maps (see methods) were averaged to obtain the mean LFP response as a function of the respiratory cycle (Fig. 1B). This averaged representation clearly revealed the breathing synchronization of gamma bursts close to the Inspiration/Expiration transition epoch.

Quantitative analysis of gamma activity was performed on gamma episodes extracted by the wavelet ridge extraction method. Each gamma burst was characterized by the amplitude, the frequency, and the respiratory phase of its maximum of energy and by its duration. The amplitude of the response in the gamma band was expressed as the ratio between the energy of the wavelet transform at each maximum and the mean energy contained in prestimulus period. Gamma episodes occurred in spindles of 40-230 ms duration (mean $85 \pm 25.7 \mathrm{~ms}$ ), a $49.39 \pm 6.86 \mathrm{~Hz}$ frequency and a normalized amplitude relative to prestimulus activity of 9.97 \pm 3.79 . Then, we analyzed gamma activity as a function of odor quality (Table 1). One-way 
ANOVA showed no statistical effect of odor quality on amplitude $(F(7,153)=1.140, p=0.34)$, duration $(\mathrm{F}(7,153)=1.379, \mathrm{p}=0.22)$ or frequency $(\mathrm{F}(7,153)=0.913, \mathrm{p}=0.496)$ of gamma bursts. From each recording, we computed the mean respiratory phase of all maxima detected in the gamma band during odor response. These mean values were used to construct the histogram of phase distribution along the respiratory cycle (Fig. 1C). The Rayleigh test for uniformity revealed that gamma burst maxima were non-uniformly distributed $(\mathrm{p}<0.0001)$ along the respiratory cycle with a mean phase of $0.54( \pm 0.073)$. Such results revealed that gamma activity was tightly phase-locked close to the inspiration-expiration (I/E) transition epoch. Comparison of circular means (Watson F tests) did not reveal statistical differences between odors (see Table 1 for details).

\section{Cell unitary responses}

As previously reported (Litaudon et al., 2003), odor stimulus evoked mainly excitatory responses in the aPC (51.4\% of total responses). A large percentage of these excitatory responses (71 \%) presented clear respiration-related modulation. In order to analyze phase relationships between spikes and respiratory activity, we computed the mean respiratory phase of spike discharge for each excitatory response. These mean values were used to construct a histogram of respiratory phase distribution (Fig. 1D). The Rayleigh test for uniformity revealed that the respiratory phases of spikes were non-uniformly distributed $(\mathrm{p}<0.0001)$, with a mean phase of $0.51( \pm 0.25)$. Comparison of spike respiratory phase distribution between odors (Watson F test) did not reveal statistical differences.

Comparison of circular means between spikes respiratory phase and gamma burst respiratory phase did not reveal statistical differences. This result showed that both spikes and gamma bursts were synchronized with the same respiratory phase, close to the I/E transition epoch. 
Phase relationship between oscillatory field potentials and unit activity

Analysis consisted of finding all spikes concomitant with a gamma burst detected by the wavelet ridge extraction method. From the time position of each spike, we found the corresponding instantaneous phase provided by the wavelet ridge (Fig. 2A). These phase values were used to construct the spike preferential instantaneous phase histogram relative to gamma oscillation. Thus, to be included in the analysis, a response should share two features: first, it should respond to odor with both spikes and detectable gamma activity; second, spikes and gamma oscillations should be concomitant. Despite these strict criteria, the analysis was performed on 87 odor responses from a total of 25 cells. Due to these conservative criteria and the low number of spikes exhibited by PC cell responses (Litaudon et al., 2003), individual cell responses could not provide reliable statistical data. In a first step, responses to all odors were pooled to construct the spike preferential instantaneous phase histograms (Fig. 2B). The significance of the phase-coupling was tested on this total histogram. The Rayleigh test for uniformity revealed that spikes were non-uniformly distributed $(\mathrm{p}<<0.0001)$ on the gamma oscillation with a mean phase -0.047 ( $\pm 1.086 \mathrm{rad}$ ), corresponding to the peak of the oscillatory wave. Moreover, the circular deviation (1.086 rad) remained small, indicating a strong phase-coupling between spikes and gamma oscillation.

In a second step, we analyzed spike/LFP phase-coupling according to odor quality (Table 2). Data showed that the mean phase of spikes relative to LFP oscillation was not statistically different between odors (Watson F tests). Such results showed that spike/LFP phase-coupling was not odor dependent.

\section{Discussion}

Data presented here describe the phase relationship between piriform cortex cell spikes and LFP fast oscillatory activity during odor evoked activity in the anesthetized rat. Our data 
showed that mean spike discharges and gamma oscillatory bursts were synchronized with the same respiratory cycle epoch (close to the inspiration/expiration transition). Temporal correlations between spikes and LFP revealed that cortical cell spikes were tightly phasecoupled with the peak of gamma oscillations.

Both single unit activity and LFP oscillatory activity were first analyzed regarding their temporal relationship with respiration. In the aPC, excitatory responses were largely entrained to the respiratory cycle as previously reported (Wilson, 1998; Litaudon et al., 2003). These respiration-synchronized responses were mainly phase-coupled around the inspiration/expiration transition epoch, as reported for olfactory bulb mitral cell activity (Buonviso et al., 2003; Buonviso et al., 2006).

Fast oscillations analysis showed that gamma activity was in the same frequency range as previously reported in both anesthetized (Neville and Haberly, 2003; Fletcher et al., 2005) and awake (Bressler, 1987; Chabaud et al., 2000) animals. The frequency, as well as the amplitude and the duration of gamma burst, did not depend on the odor identity. Such data agree with studies in the rabbit olfactory system showing that gamma amplitude maps over the OB surface seemed to be more related to the behavioral meaning of the odor than to the chemical nature of the stimulus (Freeman and Schneider, 1982; Di Prisco and Freeman, 1985; Bressler, 1988). Whereas gamma band activity was not observed during spontaneous activity, odor responses showed a clear modulation of gamma activity related to breathing, as previously described in the PC (Bressler, 1987; Fontanini and Bower, 2005) and the OB (Buonviso et al., 2003). In a previous paper, Fontanini and Bower (Fontanini and Bower, 2005) reported that spontaneous gamma activity could be observed in ketamin/xylazine anesthetized rats and that the level of respiratory modulation of gamma bursts was related to the depth of anesthesia. Such a difference from our results could be due to the difference in the anesthetic used (urethane vs ketamine/xylazine) and/or to the difference in depth of anesthesia. The absence 
of gamma band oscillation during spontaneous activity suggests that our rats were probably under deep anesthesia.

The respiratory modulation of gamma activity has already been described in several papers, but was not precisely quantified regarding its phase coupling with the different epochs (inspiration/expiration) of the respiratory cycle. Gamma bursts occurred around the I/E transition period, as observed in the OB (Buonviso et al., 2003), with a mean phase similar to spikes discharges. As previously suggested in the olfactory bulb (Buonviso et al., 2003), our data confirmed the hypothesis that the I/E transition period might be a crucial time window during which cortical structure might sample information. This respiratory period corresponded to the moment of maximal amplitude of the electro-olfactogram (Chaput, 2000), which is probably the time of maximal olfactory sensory neuron activity. As afferent connections are predominant in the aPC (Haberly, 1973; Schwob and Price, 1978), it can be hypothesized that the aPC receives large and synchronized excitation from the $\mathrm{OB}$ around the I/E transition period (Litaudon et al., 2003). Our results agree with a functional coupling between the $\mathrm{OB}$ and the aPC, as previously reported in both anesthetized (Fontanini and Bower, 2005) and awake rats (Kay and Freeman, 1998; Chabaud et al., 1999).

After showing that cortical spike discharges and gamma oscillations shared the same slow dynamic and were largely concomitant, we examined their fine-scale temporal relationships. Our data revealed that, during odor evoked activity, spikes occurring during gamma episodes were tightly coupled with the peak of the oscillation. This phase coupling was similar to the correlation between unit firing and EEG previously reported during spontaneous activity in the awake rat (Eeckman and Freeman, 1990). Such phase-coupling has also been reported in the rabbit OB (Kashiwadani et al., 1999) and insect antennal lobe (Laurent and Davidovitz, 1994), where LFP oscillations exhibit lower frequency. What is the origin of this tight phase- 
coupling? In a previous study, we proposed that gamma band oscillations could subserve a gating function for bulbo-cortical transfer of information (Buonviso et al., 2003). It has been proposed that cortical cells act as coincidence detectors to integrate OB information (Wilson and Stevenson, 2003). Indeed, previous work showed that single electrical stimulation of the LOT failed to evoked spikes in cortical cells (Ketchum and Haberly, 1993), and a recent study revealed that the integration time window of PC cells was on the order of ten milliseconds (Franks and Isaacson, 2006). The co-occurrence of gamma oscillations in the OB and the aPC may serve to constrain the firing time of neurons to narrow time windows. Oscillatory phasecoupling of spikes in the OB (Kashiwadani et al., 1999) increases the probability of spike emission in the cortical cell during short time windows, explaining the tight phase-coupling observed in the aPC. A similar mechanism of information transfer between the antennal lobe and the mushroom body has been proposed in the insect olfactory system, (Perez-Orive et al., 2002). The authors suggested that the oscillatory dynamic may serve as a temporal filter to synthesize the output from the antennal lobe.

What could the functional role of this gamma phase-coupling be? The phase of cortical neuron spikes relative to the corresponding LFP oscillation cycles varies around a mean that is stimulus independent (Table 2). Spike phase, therefore, contains no information about odor identity as reported in insect (Laurent et al., 1998). As previously explained, gamma activity could play a role of gating function for the bulbo-cortical transfer of information. Such a hypothesis received an experimental validation in a recent study showing that olfactory cortex neurons exhibited robust spike responses only during the fast wave state $(35-45 \mathrm{~Hz})$ (Murakami et al., 2005). Moreover, spike probability in PC cells increased during a brief stimulus train delivered in the LOT at gamma frequency (Franks and Isaacson, 2006). In other sensory systems, gamma activity has been proposed to play a role of binding function between stimulus features (see Singer, 1999 for review). In this way, synchronization of 
mitral/tufted cell spike discharges on the gamma oscillation may contribute to combine signals derived from different odorant receptors, and as suggested by a recent study (Zou and Buck, 2006), neurons in the aPC may act as synthetic processors. Thus, spike-gamma phasecoupling could be a mechanism allowing the binding i) between odor features detected in the olfactory bulb as proposed by Wilson and Stevenson (Wilson and Stevenson, 2003), the aPC coding for odor identity (Gottfried et al., 2006; Kadohisa and Wilson, 2006); ii) between combinations of receptor inputs, merging the receptor code of different odorants in a mixture (Zou and Buck, 2006; Yoshida and Mori, 2007). 


\section{Acknowledgements}

This work was supported by a grant from CNRS "Programme Interdisciplinaire: Cognition et Traitement de l’Information”. 


\section{References}

Bressler SL (1987) Relation of olfactory bulb and cortex. I. Spatial variation of bulbocortical interdependence. Brain Res 409:285-293.

Bressler SL (1988) Changes in electrical activity of rabbit olfactory bulb and cortex to conditioned odor stimulation. Behav Neurosci 102:740-747.

Buonviso N, Amat C and Litaudon P (2006) Respiratory modulation of olfactory neurons in the rodent brain. Chem Senses 31:145-154.

Buonviso N, Amat C, Litaudon P, Roux S, Royet JP, Farget V and Sicard G (2003) Rhythm sequence through the olfactory bulb layers during the time window of a respiratory cycle. Eur J Neurosci 17:1811-1819.

Chabaud P, Ravel N, Wilson DA and Gervais R (1999) Functional coupling in rat central olfactory pathways: a coherence analysis. Neurosci Lett 276:17-20.

Chabaud P, Ravel N, Wilson DA, Mouly A-M, Vigouroux M, Farget V and Gervais R (2000) Exposure to behaviourally relevant odour reveals differential characteristics in rat central olfactory pathways as studied through oscillatory activities. Chem Senses 25:561-573.

Chaput MA (2000) EOG responses in anesthetized freely breathing rats. Chem Senses 25:695-701.

Di Prisco GV and Freeman WJ (1985) Odor-related bulbar EEG spatial pattern analysis during appetitive conditioning in rabbits. Behav Neurosci 99:964-978.

Eeckman FH and Freeman WJ (1990) Correlations between unit firing and EEG in the rat olfactory system. Brain Res 528:238-244.

Fisher NI (1993) Statistical analysis of circular data. Cambridge University Press, Cambridge. Fletcher ML, Smith AM, Best AR and Wilson DA (2005) High-frequency oscillations are not necessary for simple olfactory discriminations in young rats. J Neurosci 25:792-798. 
Fontanini A and Bower JM (2005) Variable coupling between olfactory system activity and respiration in ketamine/xylazine anesthetized rats. J Neurophysiol 93:3573-3581.

Franks KM and Isaacson JS (2006) Strong single-fiber sensory inputs to olfactory cortex: implications for olfactory coding. Neuron 49:357-363.

Freeman WJ (1975) Mass action in the nervous system. Academic Press, New York.

Freeman WJ (1978) Spatial properties of an EEG event in the olfactory bulb and cortex. Electroencephalogr Clin Neurophysiol 44:586-605.

Freeman WJ and Schneider W (1982) Changes in spatial patterns of rabbit olfactory EEG with conditioning to odors. Psychophysiology 19:44-56.

Friedrich RW, Habermann CJ and Laurent G (2004) Multiplexing using synchrony in the zebrafish olfactory bulb. Nat Neurosci 7:862-871.

Gottfried JA, Winston JS and Dolan RJ (2006) Dissociable codes of odor quality and odorant structure in human piriform cortex. Neuron 49:467-479.

Haberly LB (1973) Summed potentials evoked in opossum prepyriform cortex. J Neurophysiol 36:775-788.

Kadohisa M and Wilson DA (2006) Separate encoding of identity and similarity of complex familiar odors in piriform cortex. Proc Natl Acad Sci U S A 103:15206-15211.

Kashiwadani H, Sasaki YF, Uchida N and Mori K (1999) Synchronized oscillatory discharges of mitral/tufted cells with different molecular ranges in the rabbit olfactory bulb. J Neurophysiol 82:1786-1792.

Kay ML and Freeman WJ (1998) Bidirectional processing in the olfactory-limbic axis during olfactory behaviour. Behav Neurosci 112:541-553.

Ketchum KL and Haberly LB (1993) Membrane currents evoked by afferent fiber stimulation in rat piriform cortex. I. Current source-density analysis. J Neurophysiol 69:248-260. 
Kronland-Martinet R, Morlet J and Grossmann A (1987) Analysis of sound patterns through wavelet transforms. Int J Patt Recogn Art Intell 1:273-302.

Laurent G and Davidovitz H (1994) Encoding of olfactory informations with oscillating neural assemblies. Science 265:1872-1875.

Laurent G, Mac leod K, Stopfer M and Wehr M (1998) Spatiotemporal structure of olfactory input to the mushroom bodies. Learn Mem 5:124-132.

Laurent G, Wehr M and Davidovitz H (1996) Temporal representations of odors in an olfactory network. J Neurosci 16:3837-3847.

Litaudon P, Amat C, Bertrand B, Vigouroux M and Buonviso N (2003) Piriform cortex functional heterogeneity revealed by cellular responses to odours. Eur J Neurosci 17:24572461.

Litaudon P and Cattarelli M (1994) Multisite optical recording of the rat piriform cortex activity. Neuroreport 5:743-746.

Litaudon P and Cattarelli M (1995) Piriform cortex late activity revealed functional spatial heterogeneity. NeuroReport 6:1377-1380.

Litaudon P, Datiche F and Cattarelli M (1997) Optical recording of the rat piriform cortex activity. Prog Neurobiol 52:485-510.

Mardia KV (1972) Statistics of directional data. Academic Press, London.

Murakami M, Kashiwadani H, Kirino Y and Mori K (2005) State-Dependent Sensory Gating in Olfactory Cortex. Neuron 46:285-296.

Neville KR and Haberly LB (2003) Beta and gamma oscillations in the olfactory system of the urethane-anesthetized rat. J Neurophysiol 90:3921-3930.

Perez-Orive J, Mazor O, Turner GC, Cassenaer S, Wilson RI and Laurent G (2002) Oscillations and sparsening of odor representations in the mushroom body. Science 297:359365. 
Roux SG, Cenier T, Garcia S, Litaudon P and Buonviso N (2007) A wavelet-based method for local phase extraction from a multi-frequency oscillatory signal. J Neurosci Meth 160:135143.

Roux SG, Garcia S, Bertrand B, Cenier T, Vigouroux M, Buonviso N and Litaudon P (2006) Respiratory cycle as time basis: an improved method for averaging olfactory neural events. J Neurosci Meth 152:173-178.

Schwob JE and Price JL (1978) The cortical projections of the olfactory bulb: development in fetal and neonatal rats with additional observations in the adult. Brain Res 151:369-374.

Singer W (1999) Neuronal synchrony: a versatile code for the definition of relations? Neuron 24:49-65.

Stopfer M, Bhagavan S, Smith BH and Laurent G (1997) Impaired odour discrimination on desynchronization of odour-encoding neural assemblies. Nature 390:70-74.

Wilson DA (1998) Habituation of odor responses in the rat anterior piriform cortex. J Neurophysiol 79:1425-1440.

Wilson DA and Stevenson RJ (2003) Olfactory perceptual learning: the critical role of memory in odor discrimination. Neurosci Biobehav Rev 27:307-328.

Yoshida I and Mori K (2007) Odorant category profile selectivity of olfactory cortex neurons. J Neurosci 27:9105-9114.

Zou Z and Buck LB (2006) Combinatorial effects of odorant mixes in olfactory cortex. Science 311:1477-1481. 
Figure legends

Figure 1 : Respiratory modulation of aPC activity. A1) Raw signal (black line) and respiratory activity (red line). Upward and downward deflections correspond to expiration (E) and inspiration (I), respectively. A2) Corresponding time-frequency representation. Solid lines and dashed lines indicate I/E and E/I transition points, respectively. The red thick line at the bottom indicates odor stimulation. The energy is normalized relative to the mean pre-stimulus activity and color coded: blue = low energy; red = high energy. B) Mean respiratory phasefrequency representation of odor evoked LFP patterns. All respiratory cycles from all responses were averaged. The energy is normalized relative to pre-stimulus activity and is color coded: blue = low energy; red = high energy. Mean respiratory signal is superimposed (black line) indicating inspiration (I) and expiration (E) epochs. C) Distribution of gamma bursts respiratory phase. For each odor evoked response, the mean respiratory phase was computed from all local maxima in the gamma band detected during odor stimulation. These mean values were used to construct the respiratory phase histogram (161 recordings). D) Distribution of cell respiratory synchronization. For each cell excitatory response, the mean respiratory phase was computed from all spikes detected during odor stimulation, and these mean values were used to build the respiratory phase histogram (162 recordings)

Figure 2: Phase relationship between aPC spikes and gamma oscillations. A) Example of single trial analysis from the raw signal displayed in figure 1A. From top to bottom: time frequency representation (A1) displaying local maximum (*) and wavelet ridge (black solid line) of a gamma oscillatory burst (enlargement of the inset in figure 1A1). The energy is normalized relative to the mean pre-stimulus activity; corresponding raw signal (A2); phase extracted along the ridge (A3) with superimposed location of spikes (open circle); (A4) spike preferential instantaneous phase histogram computed from the whole signal during odor 
stimulation (see figure 1A1) ( $\mathrm{n}=33$ spikes). The solid line represents one cycle of gamma oscillation. B) spike preferential instantaneous phase histogram relative to gamma oscillation computed from all odor responses ( $\mathrm{n}=551$ spikes). 


\section{Table legend}

Table 1: Gamma activity features as a function of odor quality

The mean amplitude, duration, frequency, and respiratory phase of gamma burst were computed for each odor. The amplitude is expressed as the ratio between gamma burst amplitude and mean prestimulus activity in the gamma band. Rayleigh test for directionality was used to test if gamma activity was non-uniformly distributed on the respiratory cycle $\left(* \mathrm{p}<10^{-5}\right)$

Table 2: Phase-coupling between gamma oscillations and spikes

The mean phase of spikes relative to gamma oscillation was computed for each odor. All spikes from all responses were pooled to compute the mean phase $( \pm S D$ ). Rayleigh test for directionality was used to test if spikes were non-uniformly distributed (phase-coupled) on the gamma oscillation $\left(* \mathrm{p}<0.001,{ }^{* *} \mathrm{p}<<0.0001\right)$. 

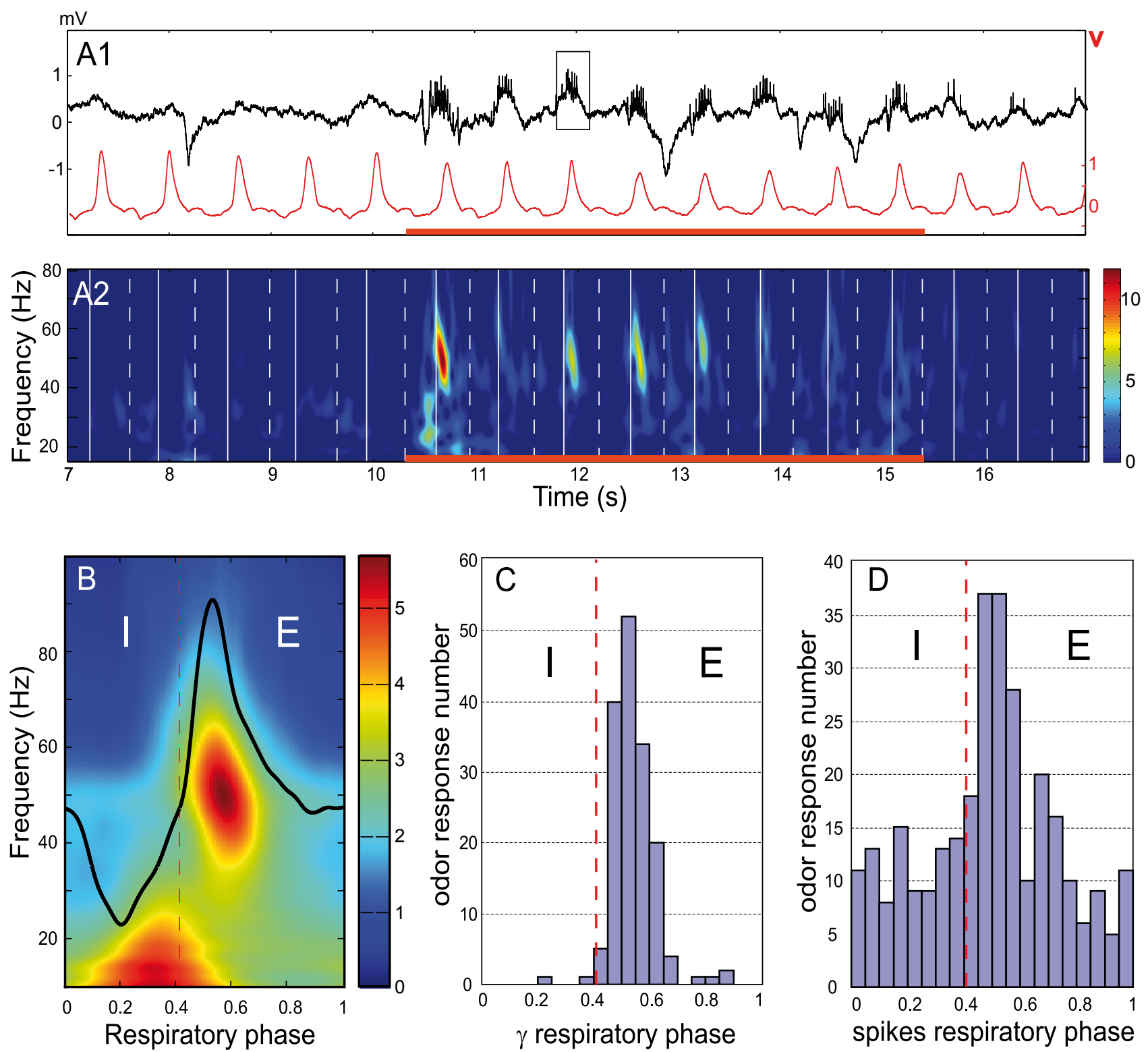

Figure 1 

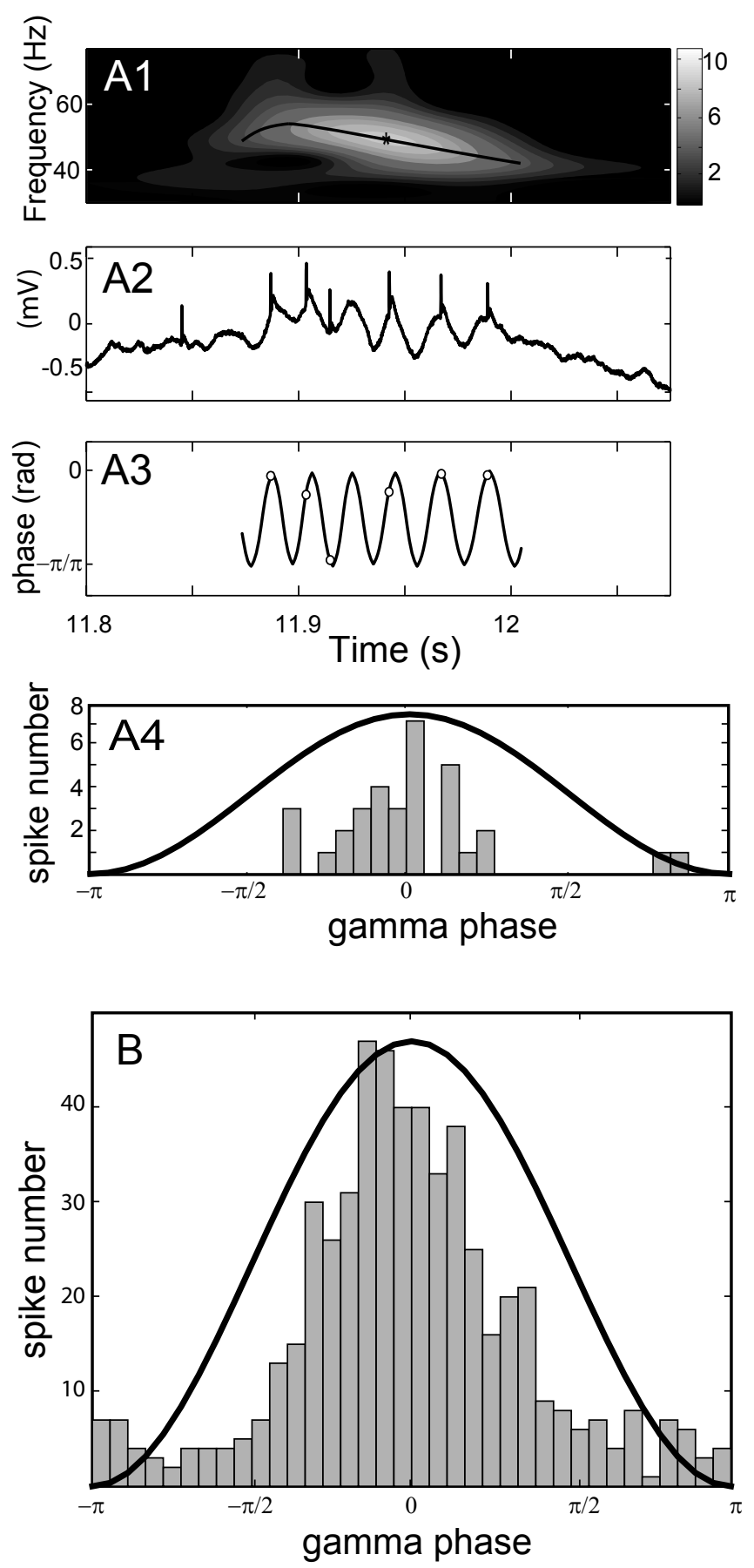

Figure 2 